\title{
Image Denoising Based on Adaptive Fractional Order Anisotropic Diffusion
}

\author{
Jimin Yu ${ }^{1}$, Lijian Tan ${ }^{1}$, Shangbo Zhou ${ }^{2}$, Liping Wang ${ }^{2}$ and Chaomei Wang ${ }^{1}$ \\ ${ }^{1}$ Chongqing University of Posts and Telecommunications \\ Chongqing, 400065 - CN \\ [e-mail: 857116721@qq.com] \\ ${ }^{2}$ Chongqing University \\ Chongqing, 400044 - CN \\ [e-mail: shbzhou@cqu.edu.cn] \\ *Corresponding author: Lijian Tan
}

Received May 17, 2016; revised October 21, 2016; accepted December 4, 2016;

published January 31, 2017

\begin{abstract}
Recently, the method based on fractional order partial differential equation has been used in image processing. Usually, the optional order of fractional differentiation is determined by a lot of experiments. In this paper, a denoising model is proposed based on adaptive fractional order anisotropic diffusion. In the proposed model, the complexity of the local image texture is reflected by the local variance, and the order of the fractional differentiation is determined adaptively. In the process of the adaptive fractional order model, the discrete Fourier transform is applied to compute the fractional order difference as well as the dynamic evolution process. Experimental results show that the peak signal-to-noise ratio (PSNR) and structural similarity index measurement (SSIM) of the proposed image denoising algorithm is better than that of other some algorithms. The proposed algorithm not only can keep the detailed image information and edge information, but also obtain a good visual effect.
\end{abstract}

Keywords: Fractional order partial differential equation, image processing, fractional differentiation, anisotropic diffusion, image texture, local variance 


\section{Introduction}

$\mathbf{I}_{\mathrm{t}}$ is known that image denoising is a significant subject in the research of image processing. Image denoising method based on integer order partial differential equations has been widely researched. This method can be divided into two categories: anisotropic diffusion denoising model proposed by Perona and Malik [1] (for short:PM denoising) and total variation proposed by Rudin, Osher and Fatemi [2] (for short: ROF denoising). The shortcomings of PM model and ROF model lie in that they are easy to lose contrasting information and texture information and produce staircase effects [3]. To solve these shortcomings, some improved algorithms are proposed. G. Motta, et al put forward an extension of the Discrete Universal DEnoiser (DUDE) which is specially used for the denoising of grayscale images [4]. Gong Yuanhao, et al put forward a variational model with local weighted Gaussian curvature as the regularizer [5]. Wang Jiefei, et al proposed a residual-based method for denoising images [6]. Very recently, Zhang Wenxue, et al proposed a $L_{0}$ gradient minimization (LGM) method for image smoothing [7]. Cui Lihong, et al proposed an extension of interscale SURE-LET approach exploiting the interscale to improve denoising performance [8].Zhao De et al proposed an improved PM model based on local entropy [9]. In order to improve the accuracy of iris recognition while dealing with non-ideal iris images, Mazhar Sajjad et al [10] proposed a novel algorithm that improves the quality of degraded iris images. Yu Hancheng et al [11] proposed a speed-up technique for the non-local means image denoising method based on local binary descriptor. Yan Zhou et al [12] proposed a new automatic underwater image enhancement algorithm, which combines nonsubsampled contoured transform domain enhancement techniques with the mechanism of the human visual system. G Ghimpeteanu, et al [13] considered an image decomposition model that provided a novel framework for image denoising. V.B.S Prasath, et al [14] proposed a fuzzy diffusion coefficient which takes into account local pixel variability for better denoising and selective smoothing of edges.

Recently, image processing based on fractional order partial differential equation has been studied in computer vision. Wang Liping, et al proposed a homotopy regularization based on fractional order total variation for image super-resolution [15]. Feng Xiangchu, et al [16] put forward a fractional order anisotropic diffusion model for image denoising, and the model not only can well suppress noise but also preserve the edge information. To select the optional order of fractional differentiation not by experiments, Che Jin, et al [17] put forward a denoising model in which the local variance was employed to express the complexity of the local image texture and the order of fractional differentiation was determined adaptively. Li $\mathrm{Bo}$, et al [18] presented a new medical image enhancement method that adjusts the fractional order according to the dynamic gradient feature of the entire image. Pu Yifei [19] put forward a set of fractional partial differential equations based on fractional total variation and fractional steepest descent approach to address the problem of traditional drawbacks of PM and ROF multi-scale denoising for texture image. Yin Xuehui, et al [20] presented a difference curvature driven fractional anisotropic diffusion for image noise removal, which uses two new techniques, fractional calculus and difference curvature, to describe the intensity variations in images. Ido Zachevsky, et al [21] proposed an algorithm for the denoising of natural images containing NST, using patchbased fractional Brownian motion model and regularization by means of anisotropic diffusion. Chen Yiming, et al [22] proposed a numerical method to estimate the variable-order fractional derivatives of an unknown signal in noisy environment. Those proposed image processing algorithms based on fractional order partial differential 
equation have made improvement on keeping detailed image information, texture information, good visual effects and image denoising.

In order to solve the problem that the optimal order of the fractional differentiation is often obtained through a large number of experiments of the image denoising process. Combining fractional differential operator with anisotropic diffusion equation, a denoising algorithm based on adaptive fractional order anisotropic diffusion is put forward. By using the local variance, the local image texture complexity adaptively is reflected and the fractional order is determined. With regard to a determined image, before image denoising we need to calculate the image local variance, to increase the computational overhead in the image denoising process. While in the frequency domain, a lot of computational overhead will be reduced and the definition of fractional order derivative of frequency domain is relatively simple. Therefore we employ Fourier transform to represent the fractional derivative, and accordingly discrete Fourier transform is used to calculate the fractional differential and the dynamic evolution process in the frequency domain. The proposed algorithm is applied in image denoising, the experimental results show that the proposed denoising algorithm can obtain a higher peak signal to noise ratio (PSNR), structural similarity index measurement (SSIM) and visual effects.

The paper is organized as follows. In Section 2, the deduction of the fractional order anisotropic diffusion denoising model is proposed. In Section 3, the realization of fractional order differential adaptive denoising model is described. In Section 4, the experimental verification and analysis is given. Finally, some conclusions are drawn in Section 5.

\section{Fractional Order Denoising Model}

\subsection{Definition of Fractional Order Differential in Frequency Domain}

In this section, we give a brief description of the fractional order derivatives in the frequency domain. The commonly used definitions are Grumwald-Letnikov definition and Riemann-Liouville definition which require to use of the Euclidean measure [23-24]. However, The definition in frequency domain is easier to implement, and we use it in this paper. Taking into account the frequency characteristic of the fractional differential, the traditional first order derivative can be extent to the fractional derivative. Some amplitude frequency characteristic curves of different fractional order differentials of $\omega$ are shown as in Fig. 1.

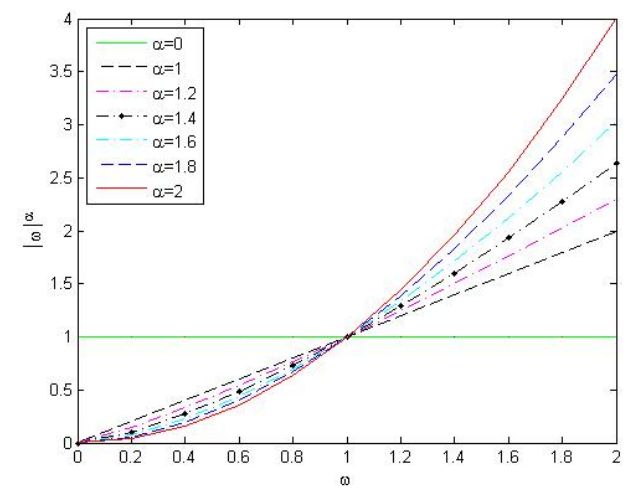

Fig. 1. Amplitude frequency characteristic curve of the fractional order differential. 
From Fig. 1, it can be obviously seen that in the low frequency part, which is corresponding to the image of the smooth region, the degree of attenuation of the fractional differential operator is similar to that of the first order differential operator. However, in the intermediate frequency and high frequency part with $\omega>1$, the fractional order differential operator is steeper than that of the first order operator. This characteristic shows that the fractional differential operator can reduce the low frequency signal and improve the high frequency signal.

For any function $f(t) \in L^{2}(R)$, it's Fourier transform is

$$
\hat{\mathrm{f}}(\omega)=\int_{R} f(t) \exp (-j \omega t) d t .
$$

Therefore, the equivalent form of fractional order differential in the frequency domain is defined as:

$$
D_{\alpha} f(t)=F^{-1}\left((j \omega)^{\alpha} \hat{f}(\omega)\right), \alpha \in R^{+},
$$

where $F^{-1}$ is the inverse operator of Fourier transform, $\alpha$ can be any real number, including decimal or fraction.

For any $g(x, y) \in L^{2}\left(R^{2}\right)$, it's corresponding two-dimensional Fourier transform is $\hat{\mathrm{g}}\left(\omega_{1}, \omega_{2}\right)=\int_{R^{2}} g(x, y) \exp \left(-j\left(\omega_{1} x+\omega_{2} y\right)\right) d x d y$. Therefore, the fractional order partial differential form with $\alpha$ is:

and

$$
D_{\alpha x} g(x, y)=F^{-1}\left(\left(j \omega_{1}\right)^{\alpha} \hat{g}\left(\omega_{1}, \omega_{2}\right)\right)
$$

$$
D_{\alpha y} g(x, y)=F^{-1}\left(\left(j \omega_{2}\right)^{\alpha} \hat{g}\left(\omega_{1}, \omega_{2}\right)\right) .
$$

\subsection{Derivation of Fractional Order Denoising Model}

\subsubsection{PM Model}

The classical PM model [1] was proposed by Perona and Malik, and its diffusion process is represented by a partial differential equation. The PM model is expressed as:

$$
\frac{\partial \mathrm{u}}{\partial t}=\operatorname{div}(c(|\nabla u|) \nabla u),
$$

where $t$ denotes the time, $c(\cdot)$ is the diffusion coefficient, $\operatorname{div}(\cdot)$ is the divergence operator, $\nabla$ is the gradient operator.

\subsubsection{ROF Model}

The classical ROF model [2] was proposed by Rudin, Osher and Fatemi, and its evolution is a nonlinear diffusion equation:

$$
\frac{\partial \mathrm{u}}{\partial t}=\operatorname{div}\left(\frac{\nabla u}{|\nabla u|}\right)-\lambda\left(u-u_{0}\right)
$$

where, $\operatorname{div}\left(\frac{\nabla u}{|\nabla u|}\right)$ is the diffusion coefficient, $\lambda\left(u-u_{0}\right)$ is the global fidelity, $\lambda(>0)$ is an adjusting parameter, for performing balanced and smooth fidelity effect. In the ROF model, 
the global fidelity of image noise is also a part of fidelity, while the ideal fidelity should only be reflected on the image edge texture details.

\subsubsection{Proposed Model}

Inspired by the literature [16] and ROF model [2], we propose the following model with anisotropic diffusion:

$$
\left\{\begin{array}{l}
\frac{\partial \mathrm{u}}{\partial t}=\operatorname{div}\left(c\left(\left|D_{\alpha} u\right|^{2}\right) D_{\alpha} u\right)+\lambda \times u \\
u(x, y, 0)=u_{0}(x, y)
\end{array},\right.
$$

where $\alpha$ denotes the fractional order, $D_{\alpha} u$ denotes the fractional derivative operator defined by $D_{\alpha} u=\left(D_{\alpha x} u, D_{\alpha y} u\right)$ and $\left|D_{\alpha} u\right|=\sqrt{D_{\alpha \mathrm{x}}^{2} u+D_{\alpha \mathrm{y}}^{2} u}, \lambda>0$ is a adjusting parameter.

By using the energy functional, we can find that the anisotropic diffusion is then shown to be an energy-dissipating process that seeks the minimum of the energy functional. We consider the following functional to be defined in the space of continuous images over the domain of $\Omega$

$$
E(u)=\int_{\Omega} f\left(\left|D_{\alpha} u\right|\right) d \Omega-\frac{\lambda}{2} u^{2},
$$

where $\Omega$ is the image region, and $\mathrm{f}(\cdot) \geq 0$ is an increasing function associated with the diffusion coefficient as $\mathrm{c}(s)=f^{\prime}(\sqrt{s}) / \sqrt{s}$. The formula (8) can be solved as the minimum value problem of the Euler-Lagrange equation, constructor functional

$$
\Phi(\mathrm{a})=\int_{\Omega} f\left(\left|D_{\alpha}(u+a \eta)\right|\right) d x d y-\frac{\lambda}{2}(u-a \eta)^{2},
$$

where test function is $\eta \in C^{\infty}(\Omega)$. To make $\Phi^{\prime}(0)=0$, and we can get

$$
\left.\int_{\Omega}\left(c\left(\left|D_{\alpha} u\right|^{2}\right) D_{\alpha x} u D_{\alpha x} \eta\right)+c\left(\left|D_{\alpha} u\right|^{2}\right) D_{\alpha y} u D_{\alpha x} \eta\right)+\lambda u \times \eta d x d y=0 .
$$

Firstly, the definition domain of $f$ is extended to $R^{2}$ from $\Omega$, based on the definition of Fourier transform of fractional derivative, and we can get the formula by using the Parseval identity $\int_{R^{2}} f \cdot g d x d y=\int_{R^{2}} \hat{f} \cdot \overline{\hat{g}} d \omega_{1} d \omega_{2}$,

$$
\left.\int_{R^{2}}\left(c\left(\left|D_{\alpha} u\right|^{2}\right) D_{\alpha x} \hat{\bar{u}} D_{\alpha x} \hat{\eta}\right)+c\left(\left|D_{\alpha} u\right|^{2}\right) D_{\alpha y} \hat{\bar{u}} D_{\alpha x} \hat{\eta}\right)+\lambda \hat{\bar{u}} \times \hat{\eta} d \omega_{1} d \omega_{2}=0 .
$$

For all functions, $\eta \in C^{\infty}(\Omega)$, it must satisfy the equation $\Phi^{\prime}(0)=0$, so there has to be

$$
D_{\alpha x}^{*}\left(c\left(\left|D_{\alpha} u\right|^{2}\right) D_{\alpha x} u\right)+D_{\alpha y}^{*}\left(c\left(\left|D_{\alpha} u\right|^{2}\right) D_{\alpha y} u\right)+\lambda \times u=0,
$$

where $D_{\alpha x}^{*}$ is the adjoint of $D_{\alpha x}, D_{\alpha y}^{*}$ is the adjoint of $D_{\alpha y}$.Thus, the Euler-Lagrange equation of the formula (8) can be rewritten as formula (12), in which the time variable is introduced. The formula (12) may be solved through the following gradient descent procedure:

$$
\frac{\partial u}{\partial t}=-D_{\alpha x}^{*}\left(c\left(\left|D_{\alpha} u\right|^{2}\right) D_{\alpha x} u\right)-D_{\alpha y}^{*}\left(c\left(\left|D_{\alpha} u\right|^{2}\right) D_{\alpha y} u\right)-\lambda \times u .
$$

This solution is arrived when $t \rightarrow \infty$, but the evolution is likely to stop earlier to achieve an 
optimal trade-off between noise removal and boundary preservation.

\section{Realization of Adaptive Fractional Differential Denoising Model}

\subsection{Construction of Adaptive Fractional Order Differential Operator}

A large number of experiments show that in the process of image denoising, when the order $\alpha$ of fractional differential is greater than 1 , the "staircase effect" can be effectively suppressed, but it is too large to suppress noise. Therefore, for an image that the texture complexity of each region is not the same, we should choose different order of fractional order differential, in order to achieve better denoising effects. The local variance can well reflect regional texture complexity, for the local variance of the region with complex texture is relatively large, and that of the region with simple texture is small [17]. In this way, it is devoted to find a function mapping between the order of fractional order differential and the local variance of the image, and we can adaptively generate the order of fractional order differential based on the local variance of an image.

We suppose that the local mean and local variance of each pixel in the noise image $u(x, y)$ are respectively $\mu_{x, y}$ and $\sigma_{x, y}$, and $M$ is a $m_{1} \times m_{2}$ rectangular window with $(x, y)$ as the center, then the following formulas can be got:

$$
\mu_{x, y}=\frac{1}{m_{1} \times m_{2}} \sum_{x, y \in M} u(x, y), \sigma_{x, y}=\frac{1}{m_{1} \times m_{2}} \sum_{x, y \in M}\left(u^{2}(x, y)-\mu^{2}(x, y)\right) .
$$

According to the form of the local entropy function proposed in [9], we construct a relation function between the local variance function and the adaptive fractional order as:

$$
\text { Adaptive fractional order }=f(\sigma)=k_{0}+k_{1} \times \frac{\sigma_{x, y}-\min \left(\sigma_{x, y}\right)}{\max \left(\sigma_{x, y}\right)-\min \left(\sigma_{x, y}\right)},
$$

where $k_{0}, k_{1}$ is the coefficient, $\sigma_{x, y}$ denotes the variance, $\min \left(\sigma_{x, y}\right)$ denotes the minimum variance, $\max \left(\sigma_{x, y}\right)$ denotes the maximum variance. The denoising results of an image with formula (14) as the adaptive operator are shown as in Fig. 2, where $k_{0}=1, k_{1}=0.693$, the variance of the Gaussian white noise is $\delta=15, \delta=30$.

Further more, exponential function is an increasing function in the real number field, and the degree of enhancement will depend on the increasing of the independent variable, which is consistent with the change of the order of the fractional differential [25-26], so we make the relationship formula between the local variance function and the adaptive fractional order as

$$
\alpha=e^{f(\delta)}+k_{2},
$$

where $k_{0}=0, k_{2}$ is the adjusting parameter. Fig. 3 shows the denoising results with formula (15) as the adaptive operator, where $k_{1}=0.693, k_{2}=0.5$.

Table 1 lists the comparison results of PSNR, SSIM and information entropy (ENTROY) between formula (14) and formula (15) as the adaptive operator, the variance of the Gaussian white noise is $\delta=15$. 


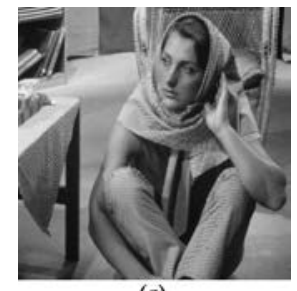

(a)
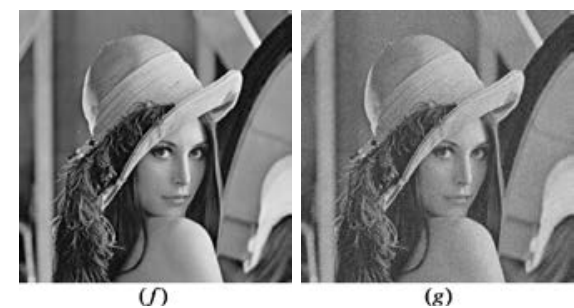

(g)

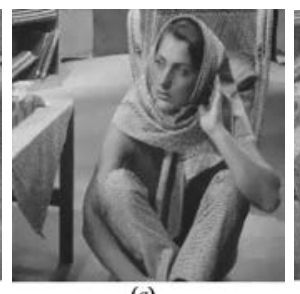

(c)

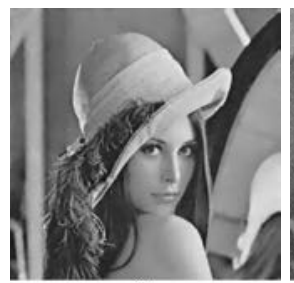

(h)

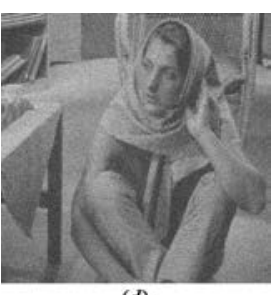

$(d)$

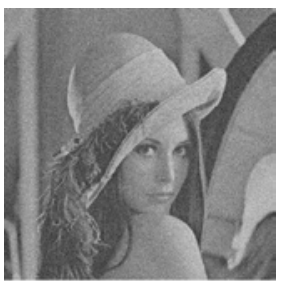

(i)

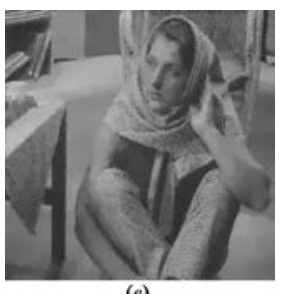

(e)

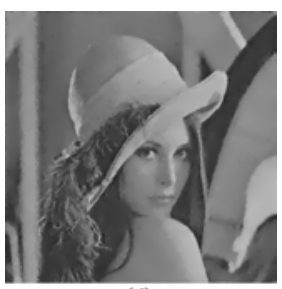

$(j)$

Fig. 2. Denoising image with formula (14) as an adaptive operator. (a) original Barbara image,(b) noisy Barbara image, $\delta=15$,(c) denoised Barbara image, $\delta=15$,(d) noisy Barbara image, $\delta=30$,

(e) denoised Barbara image, $\delta=30$,(f) original Lena image,(g) noisy Lena image, $\delta=15$, (h) denoised Lena image, $\delta=15$,(i) noisy Lena image, $\delta=30$,(j) denoised Lena image, $\delta=30$

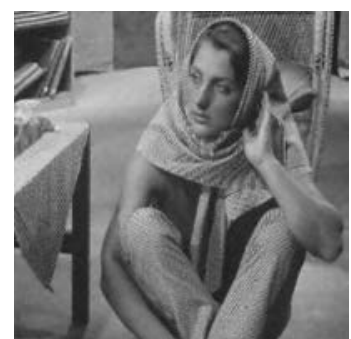

(a)

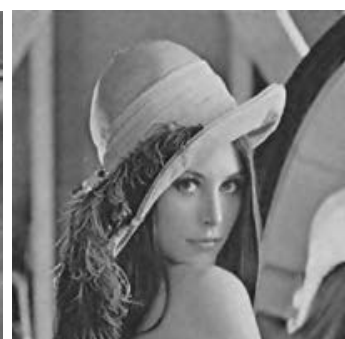

(b)

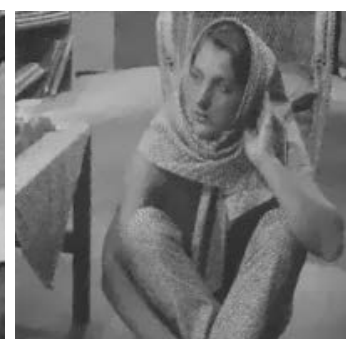

(c)

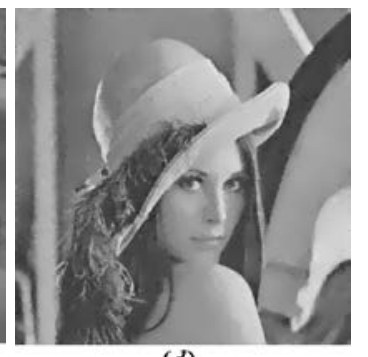

(d)

Fig. 3. Denoising images with formula (15) as an adaptive operator. (a) denoised Barbara image, $\delta=15$,(b) denoised Lena image, $\delta=15$,(c) denoised Barbara image, $\delta=30$,(d) denoised Lena image, $\delta=30$

Table 1. The comparison results of PSNR, SSIM and information entropy (ENTROY) between formula (14) and formula (15), the variance of the Gaussian white noise is $\delta=15$

\begin{tabular}{|c|c|c|c|c|c|c|c|}
\hline \multirow{2}{*}{ Image } & \multicolumn{2}{|c|}{ PSNR } & \multicolumn{2}{c|}{ SSIM } & \multicolumn{2}{c|}{ ENTROY } & \multirow{2}{*}{ The original image } \\
& EqT.(14) & Eq.(15) & Eq.(14) & Eq.(15) & Eq.(14) & Eq.(15) & ENTOY \\
\hline \hline Barbara & 28.9037 & 29.1882 & 0.8452 & 0.8595 & 7.4535 & 7.4740 & 7.4664 \\
\hline Lena & 31.5229 & 32.3395 & 0.8445 & 0.8612 & 7.4168 & 7.4459 & 7.4455 \\
\hline
\end{tabular}

From the subjective feelings of Fig. 2 and Fig. 3, and the objective reflection of the data in Table 1, we can see that the values of PSNR, SSIM and ENTROY of the denoising image with formula (15) are higher than that of formula (14). That implies that formula (15) is able to reflect the corresponding relationship better than formula (14) between the local variance of the image and the adaptive fractional order.

\subsection{Numerical Implementation of the Adaptive Denoising Model}

For practical applications, we assume that the pixel of the original discrete image $u$ is $m \times m$. 
The discrete Fourier transform is easily to implement and its computational cost is very low. In addition, it is consistent with the continuous Fourier transform which is used to calculate the fractional order differential, therefore by two-dimensional discrete Fourier transform (2-D DFT), the fractional order differential of the formula (13) can be calculated.

By the definition of the first order partial differential:

$$
D_{x} u=\frac{u(x, y)-u(x-\Delta x, y)}{\Delta x},
$$

we know that the shortest distance of two dimensional digital image signal intensity changes is between two adjacent pixels, so the duration of two-dimensional digital image on the direction of $x$ axis and $y$ axis could only be measured in pixel units, the smallest equal interval of $u(x, y)$ can only be $\Delta x=\Delta y=1$ [27], so $D_{x} u=u(x, y)-u(x-1, y)$.

Therefore, based on the translation property of the two-dimensional discrete Fourier transform, and the equivalent form of the fractional order partial differential in the frequency domain can be obtained:

$$
D_{\alpha x} u \leftrightarrow\left(1-\exp \left(-j 2 \pi \omega_{1} / m\right)\right)^{\alpha} \hat{u}\left(\omega_{1}, \omega_{2}\right),
$$

where, the fractional order $\alpha$ is the adaptive fractional order.

In the actual calculation, the central difference method is used to calculate the fractional order differential. In order to keep consistency with the two-dimensional continuous Fourier transform, the two-dimensional discrete Fourier transform operator is expressed by $F$, and the inverse operator of the two-dimensional discrete Fourier transform is represented by $F^{-1}$, so we have:

$$
\tilde{D}_{\alpha x} u=F^{-1}\left(\left(1-\exp \left(-j 2 \pi \omega_{1} / m\right)\right)^{\alpha} \times \exp \left(j \pi \alpha \omega_{1} / m\right) F(u)\right) .
$$

Now, we discuss how to calculate the conjugate matrix of $\tilde{D}_{\alpha x} u$. Let $K_{1}$ be the pure diagonal matrix in the frequency domain, that is:

$K_{1}=\operatorname{diag}\left(\left(1-\exp \left(-j 2 \pi \omega_{1} / m\right)\right)^{\alpha} \times \exp \left(j \pi \alpha \omega_{1} / m\right)\right)$. We get $\tilde{D}_{\alpha x}=F^{-1} \circ K_{1} \circ F$. Let $\tilde{D}_{\alpha x}^{*}$ represent the conjugate matrix of $\tilde{D}_{\alpha x}$, and the conjugate matrix of $F$ is $F^{-1}$, we get $\tilde{D}_{\alpha x}^{*}=F^{-1} \circ K_{1}^{*} \circ F$. Because $K_{1}$ is a pure diagonal matrix, $K_{1}^{*}$ represents the complex conjugate matrix of $K_{1}$, $\operatorname{conj}(\cdot)$ represents the complex conjugate operator, we have

$$
\tilde{D}_{\alpha x}^{*}=F^{-1}\left(\operatorname{conj}\left(\left(1-\exp \left(-j 2 \pi \omega_{1} / m\right)\right)^{\alpha} \times \exp \left(j \pi \alpha \omega_{1} / m\right)\right) F(u)\right) .
$$

By the same way, $\tilde{D}_{\alpha y}$ and $\tilde{D}_{\alpha y}^{*}$ also can be calculated.

Since $\tilde{D}_{\alpha x}, \tilde{D}_{\alpha x}^{*}, \tilde{D}_{\alpha y}$ and $\tilde{D}_{\alpha y}^{*}$ are obtained, the denoising algorithm can be obtained as follows:

$$
\hat{\mathrm{g}}=K_{1}^{*} \circ F\left(c\left(\left|\tilde{D}_{\alpha} u\right|^{2}\right) \tilde{D}_{\alpha x} u\right)+K_{2}^{*} \circ F\left(c\left(\left|\tilde{D}_{\alpha} u\right|^{2}\right) \tilde{D}_{\alpha y} u\right)+\lambda \times u,
$$

where $\tilde{D}_{\alpha} u=\left(\tilde{D}_{\alpha x} u, \tilde{D}_{\alpha y} u\right), K_{1}^{*}=\operatorname{diag}\left(\operatorname{conj}\left(\left(1-\exp \left(-j 2 \pi \omega_{1} / m\right)\right)^{\alpha} \times \exp \left(j \pi \alpha \omega_{1} / m\right)\right)\right)$, $K_{2}^{*}=\operatorname{diag}\left(\operatorname{conj}\left(\left(1-\exp \left(-j 2 \pi \omega_{2} / m\right)\right)^{\alpha} \times \exp \left(j \pi \alpha \omega_{2} / m\right)\right)\right)$, $c\left(\left|\tilde{D}_{\alpha} u\right|^{2}\right)=\frac{1}{1+\left|\tilde{D}_{\alpha x} u\right|^{2}+\left|\tilde{D}_{\alpha y} u\right|^{2}}$.

In the process of image denoising, the peak signal to noise ratio is used to be the evaluation 
criterion of the experimental results of denoising images. The peak signal to noise ratio is defined as:

$$
M S E=\frac{1}{M \times N} \sum_{i=1}^{M} \sum_{j=1}^{N}\left[u_{\mathrm{n}}(i, j)-u_{0}(i, j)\right]^{2}, \quad P S N R=10 \lg \frac{255^{2}}{M S E},
$$

where, the size of the original image is $M \times N, u_{0}(i, j)$ represents the original image, and $u_{\mathrm{n}}(i, j)$ represents the denoising image.

According to formula (13) to calculate evolution image of noise images $u(x, y)$, which were solved by the iterative method, the calculation steps are as follows:

(1) Input parameter: $u(x, y)$ is the noise image;

(2) Initialization: set $n=1, u_{n}(x, y)=u(x, y), k, \Delta t=0.1, t=k \times \Delta t, \quad k_{1}=0.693$, $k_{2}=0.5, \lambda=e^{-60}$;

(3) Compute the local mean and variance of the noise image $u(x, y)$, and then the order of the fractional differential operator is obtained by the formula (15);

(4) Compute the two dimensional discrete Fourier transform $\hat{u}_{n}(x, y)$ of $u_{n}(x, y)$, calculate the partial differential $\tilde{D}_{\alpha x} u_{n}(x, y)$ and $\tilde{D}_{\alpha y} u_{n}(x, y)$ with formula (18);

(5) Compute $\hat{u}_{n+1}(x, y)$, with iteration method to get $\hat{u}_{n+1}(x, y)=\hat{u}_{n}(x, y)-\Delta t \times \hat{g}_{n}(x, y)$;

(6) Judge whether the peak signal to noise ratio (PSNR) is the maximum or not. If not, appointing $n=n+1$, and then turning to step (5); if so, stop the iteration and output the result.

\section{Experimental Verification and Analysis}

In this section, we will verify the effectiveness of the proposed adaptive fractional order denoising algorithm, by comparing with Gaussian filtering, the fractional order PM model (pseudo-PDEs) proposed by Bai Jian et al [16], the curvature filtering (Curvature Filter) proposed by Gong Yuanhao et al [5], an adaptive TV model (Adaptive TV Mode) proposed by Che Jin [17], Zhao De et al proposed an improved PM model based on local entropy (ZHAO Model) [9] and V.B.S Prasath et al [14] proposed a denoising model based on fuzzy diffusion coefficient(FDC Model). In addition, the numerical iterative process will stop at the point where peak signal-to-noise ratio is the biggest. We have implemented simulation here in MATLAB R2012a on a computer with 2.40GHz Intel Core i7 with 4GB RAM.

Selection of images with different textures with size $512 \times 512$ including Barbara image, Metallographic image, Lena image and Satellite image. We add the Gaussian white noise with variance of $\delta=15$ into the original image, the four sets of noise images are denoised using adaptive fractional order denoising model proposed in this paper, Gaussian filtering, pseudo-PDEs, Curvature Filter, Adaptive TV Mode and ZHAO model, and the denoising images of noise image(b) processing are shown respectively as in Fig. 4, Fig. 5, Fig. 6, Fig.7, the comparison results of the PSNR, the differences of the SSIM, the comparison results of the ENTROY of the denoising images are respectively shown as in Table 2, Table 3 and Table 4, the comparison results of the running time of these different types of algorithms shown as in Table 5. 

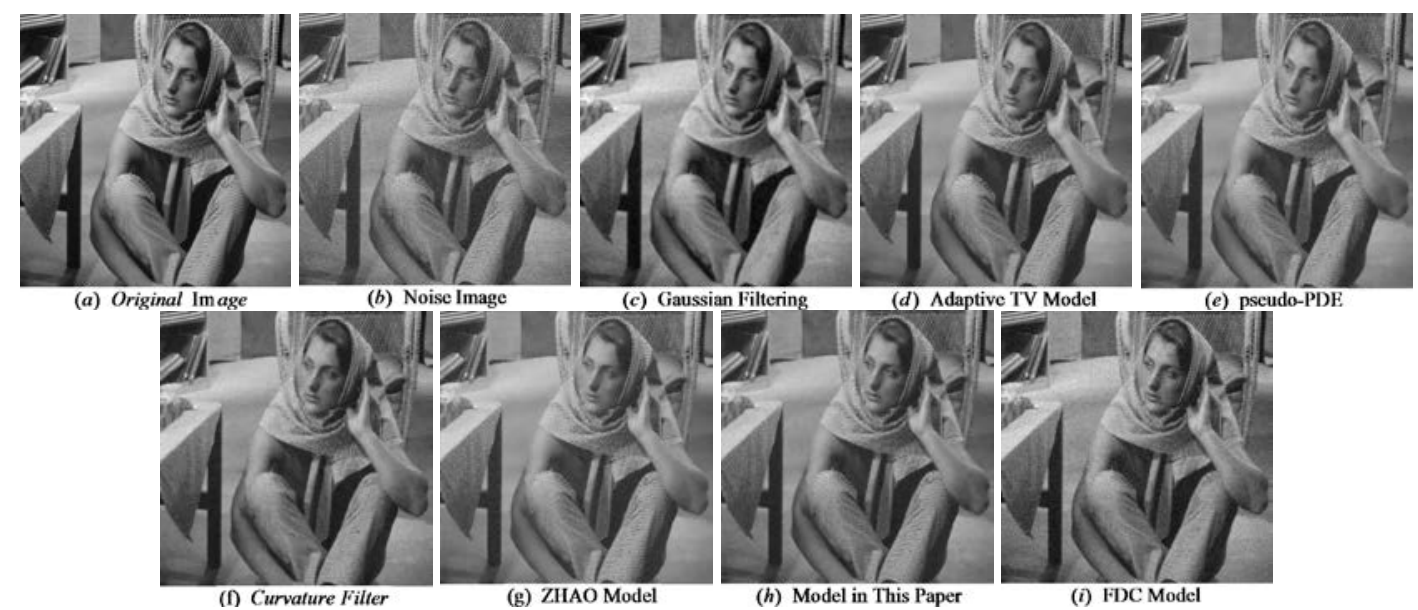

Fig. 4. Denoising images of noisy Barbara image (b) with different types of algorithms

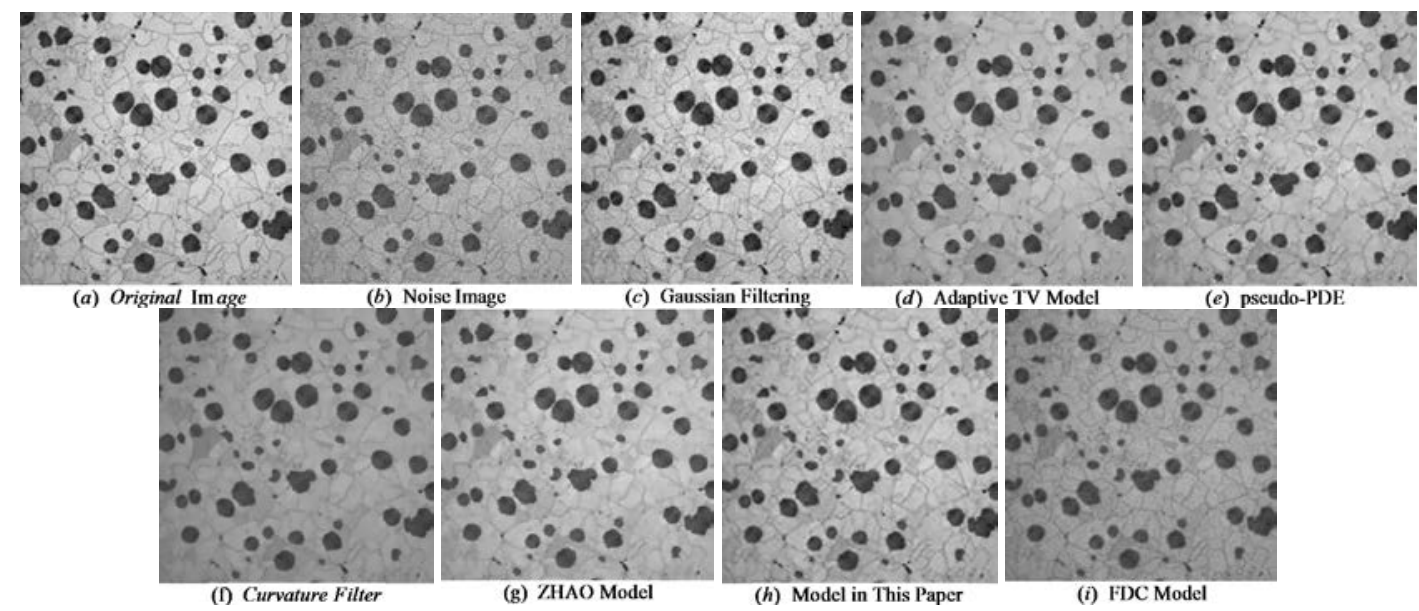

Fig. 5. Denoising images of noisy Metallographic image (b) with different types of algorithms
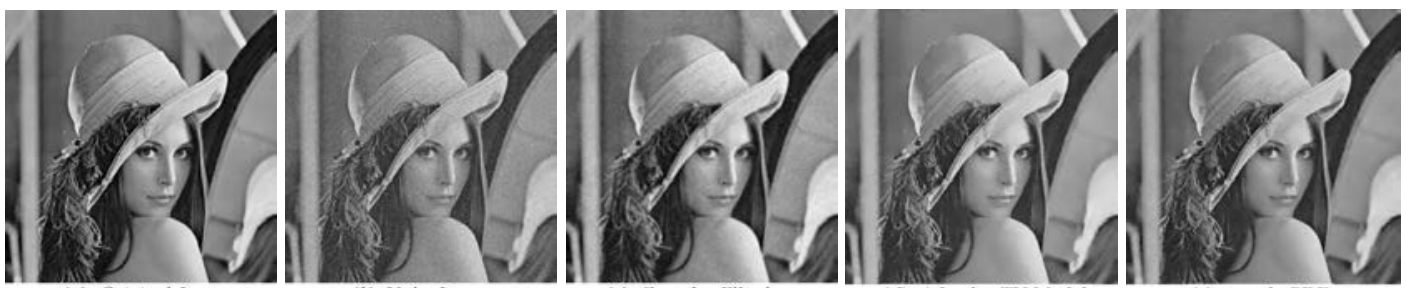

(a) Original Image
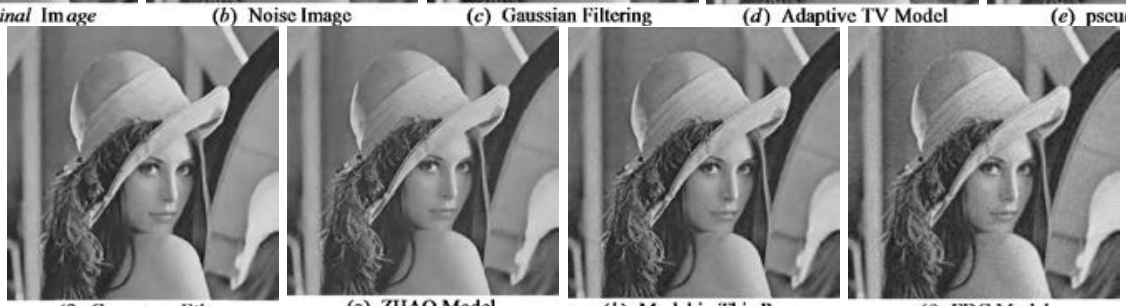

Fig. 6. Denoising images of noisy Lena image (b) with different types of algorithms 

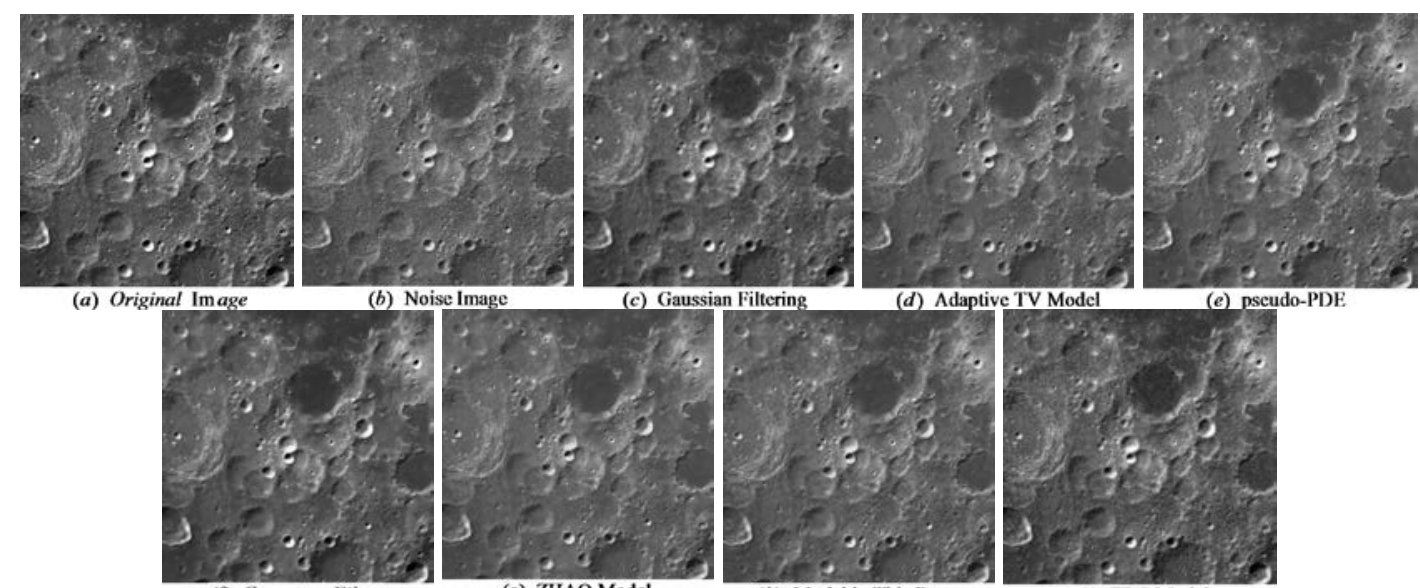

(f) Curvature Filter

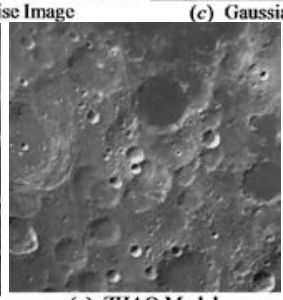

(g) ZHAO Model

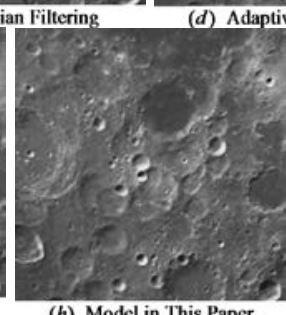

$\begin{array}{llll}\text { Fig. 7. Denvature Filter } & \text { (g) ZHAO Model } & (h) \text { Model in This Paper } & (i) \text { FDC Model } \\ \text { Denoising images of noisy Satellite image (b) with different types of algorithms }\end{array}$

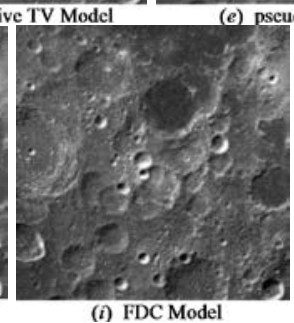

Table 2. Comparison results of the PSNR of the denoising image with different types of algorithms

\begin{tabular}{|c|c|c|c|c|c|c|c|}
\hline Image & $\begin{array}{c}\text { Gaussian } \\
\text { filtering }\end{array}$ & $\begin{array}{c}\text { Adaptive } \\
\text { TV Model }\end{array}$ & $\begin{array}{c}\text { Pseudo- } \\
\text { PDEs }\end{array}$ & $\begin{array}{c}\text { Curvatur } \\
\text { e Filter }\end{array}$ & $\begin{array}{c}\text { ZHAO } \\
\text { Model }\end{array}$ & $\begin{array}{c}\text { FDC } \\
\text { Model }\end{array}$ & $\begin{array}{c}\text { Model in } \\
\text { This Paper }\end{array}$ \\
\hline \hline Barbara & 24.1118 & 28.926 & 28.9968 & 27.4634 & 27.8251 & 28.6852 & $\mathbf{2 9 . 1 8 8 2}$ \\
\hline Metallographic & 33.667 & 32.2639 & 33.063 & 33.261 & 31.6419 & 31.5397 & $\mathbf{3 3 . 8 9 5 5}$ \\
\hline Lena & 30.197 & 31.505 & 31.9991 & 32.172 & 30.6552 & 30.9927 & $\mathbf{3 2 . 3 3 9 5}$ \\
\hline Satellite & 29.6437 & 29.6835 & 30.1351 & 29.9147 & 28.6496 & 29.2473 & $\mathbf{3 0 . 4 5 4 4}$ \\
\hline
\end{tabular}

Table 3. Comparison results of the SSIM of the denoising image with different types of algorithms

\begin{tabular}{|c|c|c|c|c|c|c|c|}
\hline Image & $\begin{array}{c}\text { Gaussian } \\
\text { filtering }\end{array}$ & $\begin{array}{c}\text { Adaptive } \\
\text { TV Model }\end{array}$ & $\begin{array}{c}\text { Pseudo- } \\
\text { PDEs }\end{array}$ & $\begin{array}{c}\text { Curvatur } \\
\text { e Filter }\end{array}$ & $\begin{array}{c}\text { ZHAO } \\
\text { Model }\end{array}$ & $\begin{array}{c}\text { FDC } \\
\text { Model }\end{array}$ & $\begin{array}{c}\text { Model in } \\
\text { This Paper }\end{array}$ \\
\hline \hline Barbara & 0.6707 & 0.8263 & 0.8522 & 0.8073 & 0.8086 & 0.6883 & $\mathbf{0 . 8 5 9 5}$ \\
\hline Metallographic & 0.9094 & 0.8667 & 0.8570 & 0.8651 & 0.8274 & 0.7762 & $\mathbf{0 . 8 7 1 2}$ \\
\hline Lena & 0.8270 & 0.8425 & 0.8569 & 0.8582 & 0.8319 & 0.8322 & $\mathbf{0 . 8 6 1 2}$ \\
\hline Satellite & 0.8045 & 0.8007 & 0.8082 & 0.7946 & 0.7559 & 0.7911 & $\mathbf{0 . 8 1 9 3}$ \\
\hline
\end{tabular}

Table 4. Comparison results of the ENTROY of the denoising image with different types of algorithms

\begin{tabular}{|c|c|c|c|c|c|c|c|}
\hline Image & $\begin{array}{c}\text { Gaussian } \\
\text { filtering }\end{array}$ & $\begin{array}{c}\text { Adaptive } \\
\text { TV Model }\end{array}$ & $\begin{array}{c}\text { Pseudo- } \\
\text { PDEs }\end{array}$ & $\begin{array}{c}\text { Curvatur } \\
\text { e Filter }\end{array}$ & $\begin{array}{c}\text { ZHAO } \\
\text { Model }\end{array}$ & $\begin{array}{c}\text { FDC } \\
\text { Model }\end{array}$ & $\begin{array}{c}\text { Model in } \\
\text { This Paper }\end{array}$ \\
\hline \hline Barbara & 7.3886 & 7.4606 & 7.4728 & 7.4308 & 7.5315 & 7.4228 & $\mathbf{7 . 4 7 4 0}$ \\
\hline Metallographic & 6.3995 & 6.3973 & 6.3829 & 6.2256 & 6.3038 & 6.3737 & $\mathbf{6 . 4 6 5 3}$ \\
\hline Lena & 7.4220 & 7.0379 & 7.4342 & 7.3757 & 7.4382 & 7.4368 & $\mathbf{7 . 4 4 5 9}$ \\
\hline Satellite & 6.8025 & 6.8602 & 6.8632 & 6.7904 & 6.8498 & 6.6550 & $\mathbf{6 . 8 8 6 4}$ \\
\hline
\end{tabular}

Table 5. Comparison results of the running time of these different types of algorithms(Unit: Second)

\begin{tabular}{|c|c|c|c|c|c|c|c|}
\hline Image & $\begin{array}{c}\text { Gaussian } \\
\text { filtering }\end{array}$ & $\begin{array}{c}\text { Adaptive } \\
\text { TV Model }\end{array}$ & $\begin{array}{c}\text { Pseudo- } \\
\text { PDEs }\end{array}$ & $\begin{array}{c}\text { Curvatur } \\
\text { e Filter }\end{array}$ & $\begin{array}{c}\text { ZHAO } \\
\text { Model }\end{array}$ & $\begin{array}{c}\text { FDC } \\
\text { Model }\end{array}$ & $\begin{array}{c}\text { Model in } \\
\text { This Paper }\end{array}$ \\
\hline \hline Barbara & 0.9359 & 118.4912 & 182.2906 & 37.1060 & 13.7582 & 30.6762 & $\mathbf{2 4 7 . 8 0 9 7}$ \\
\hline Metallographic & 1.3746 & 114.8895 & 174.4096 & 45.1872 & 13.5501 & 809.6865 & $\mathbf{2 4 1 . 2 4 0 5}$ \\
\hline Lena & 0.9418 & 123.8183 & 191.5609 & 47.4400 & 14.1970 & 256.3321 & $\mathbf{2 5 7 . 1 6 2 1}$ \\
\hline Satellite & 0.9526 & 114.9078 & 196.6252 & 44.2217 & 13.3099 & 184.6692 & $\mathbf{2 4 3 . 6 0 1 2}$ \\
\hline
\end{tabular}

We can carry on the objective evaluation for the image edge enhancement and image weak texture retention, by analyzing the peak signal to noise ratio, the information entropy and the similarity of the image. PSNR can represent the image noise removal effects, from Table 2, it can be seen that the values of the adaptive fractional order algorithm proposed in this paper is 
highest, and the image of the overall denoising effect is better. Comparing with table of information entropy data, it can be seen that the image dealt with denoising algorithm and the original image information entropy are relatively close, it shows that the images have remained some texture information after denoising. SSIM is mainly used for comparing the degree of similarity between the two images, the higher the value, the more similar image content is. From Table 3, the value of SSIM of each denoising algorithm is similar, which can remove the noise while retaining the original content of the image. Meanwhile, the value of SSIM of the proposed adaptive fractional order algorithm is slightly higher, which has better texture details in the image, and has a good visual effect. From Table 5, we can see that the running time of the algorithm proposed in this paper is the longest, the reason is that the algorithm requires calculate the image local variance before the image denoising, thus increasing the computational overhead of image denoising process, increasing the computation time of the image.

\section{Conclusion}

In this paper, an image denoising algorithm based on adaptive fractional order anisotropic diffusion is proposed. The local variance is employed to represent the local image texture complexity and the fractional order is determined adaptively. It is not only beneficial to the fractional order partial differential operator in the practical application of promotion, but also can further improve the fractional partial differential operator to the ability of noise and it has laid a good foundation for the subsequent image processing. With the comparison results, it can be seen that the proposed image denoising algorithm can effectively overcome the drawbacks of losing contrasting information and texture information. It can achieve improvement on keeping detailed image information, texture information, good visual effects, better PSNR and SSIM in the process of image denoising.

\section{Acknowledgments}

This work was supported by the major project of Fundamental Science and Frontier Technology Research of Chongqing CSTC (Grant No.cstc2015jcyjBX0124), Chongqing People's Livelihood Science and technology innovation projects (Grant No. cstc2015shmszx120010).

\section{References}

[1] P Perona, J Malik, "Scale-space and edge detecting using anisotropic diffusion," IEEE Transactions on Pattern Analysis \& Machine Intelligence, vol. 12, no. 7, pp. 629-639, July, 1990. Article (CrossRef Link)

[2] L Rudin, S Osher, E Fatemi, "Nonlinear total variation based noise removal algorithms," Physica D-Nonlinear Phenom, vol. 60, no. 1-4, pp. 259-268, 1992. Article (CrossRef Link)

[3] T Chan, S Esedoglu, Park F, Recent developments in total variation image restoration, 1nd Edition, New York, 2005. Article (CrossRef Link)

[4] Giovanni Motta, "The iDUDE framework for grayscale image denoising," IEEE Transactions on Image Processing, vol. 20, no. 1, pp. 1-21, September, 2011. Article (CrossRef Link)

[5] Gong Yuanhao, Sbalzarini Ivo F., "Local weighted Gaussian curvature for image processing," in Proc. of 20th IEEE International Conference on Image Processing, pp. 534-538, September 15-18, 2013. Article (CrossRef Link) 
[6] Wang Jiefei, Chen Yupeng, Li Tao, Lu Jian,and Shen Lixin, “A residual-based kernel regression method for image denoising,” Mathematical Problems in Engineering, pp. 1-13, March, 2016. Article (CrossRef Link)

[7] Zhang Wenxue, Cao Yongzhen, Zhang Rongxin, Li Lingling, and Wen Yunlei, "Image denoising via $L_{0}$ gradient minimization with effective fidelity term," Mathematical Problems in Engineering, pp. 1-11, December, 2015. Article (CrossRef Link)

[8] Cui Lihong, Wang Zhan, Cen Yigang, "An extension of the interscale SURE-LET approach for image denoising,” International Journal of Advanced Robotic Systems, vol. 11, no. 1, pp. 257-267, January, 2014. Article (CrossRef Link)

[9] Zhao De, He Chuanjiang, and Chen Qiang, “Anisotropic diffusion model combined with local entropy,” Pattern Recognition and Artificial Intelligence, vol. 25, no. 4, pp. 642-647, April, 2012. Article (CrossRef Link)

[10] Sajjad Mazhar, Ahn Chang-Won, Jung Jin-Woo, "Iris image enhancement for the recognition of non-ideal iris images,” KSII Transactions on Internet and Information Systems, vol. 10, no. 4, pp. 1904-1926, April, 2016. Article (CrossRef Link)

[11] Yu Hancheng, Li Aiting, "Real-time non-local means image denoising algorithm based on local binary descriptor,” KSII Transactions on Internet and Information Systems, vol. 10, no. 2, pp. 825-836, February, 2016. Article (CrossRef Link)

[12] Zhou Yan, Li Qingwu, Huo Guanying, "Human visual system based automatic underwater image enhancement in NSCT domain,” KSII Transactions on Internet and Information Systems, vol. 10, no. 2, pp. 837-856, February, 2016. Article (CrossRef Link)

[13] G Ghimpeţeanu, T Batard, M Bertalmío, "A decomposition framework for image denoising algorithms,” Image Processing IEEE Transactions on, vol. 25, no. 1, pp. 388-399, January, 2016. Article (CrossRef Link)

[14] VBS Prasath, R Delhibabu, "Image restoration with fuzzy coefficient driven anisotropic diffusion," in Proc. of 5th International Conference on Swarm, Evolutionary, and Memetic Computing, pp. 145-155, December 18-20, 2015. Article (CrossRef Link)

[15] Wang Liping, Zhou Shangbo, and Karim Awudu, "Super-resolution image reconstruction method using homotopy regularization,” Multimed Tools Applications, pp. 1-24, September, 2015. Article (CrossRef Link)

[16] Bai Jian , Feng Xiangchu, "Fractional-order anisotropic diffusion for image denoising," IEEE Transactions on Image Processing, vol. 16, no. 10, pp. 2492-2502, October, 2007. Article (CrossRef Link)

[17] Che Jin, Guan Qian, and Wang Xiyuan, "Image denoising based on adaptive fractional partial differential equations,” in Proc. of 6th International Congress on Image and Signal Processing, pp. 288-292, December 16-18, 2013. Article (CrossRef Link)

[18] Li Bo, Xie Wei, "Adaptive fractional differential approach and its application to medical image enhancement,” Computers and Electrical Engineering, vol. 45, pp. 324-335, July, 2015. Article (CrossRef Link)

[19] Pu Yifei, Slarry Patrick, and Zhou Jiliu, "Fractional partial differential equation denoising models for texture image,” Science China Information Sciences, vol. 57, no. 7, pp. 1-19, May, 2014. Article (CrossRef Link)

[20] Yin Xuehui, Zhou Shangbo, "Image structure-preserving denoising based on difference curvature driven fractional nonlinear diffusion,” Mathematical Problems in Engineering, pp. 1-16, April, 2015. Article (CrossRef Link)

[21] Zachevsky, Ido, Zeevi, Yehoshua Y, "Statistics of natural stochastic textures and their application in image denoising,” IEEE Transactions on Image Processing, vol. 25, no. 2, pp. 2130-2145, May, 2016. Article (CrossRef Link)

[22] Chen Yiming, Wei Yanqiao, Liu Dayan, "Variable-order fractional numerical differentiation for noisy signals by wavelet denoising,” Journal of computational physics, vol. 311, pp. 338-347, April, 2016. Article (CrossRef Link)

[23] KB Oldham, J Spanier, “The fractional calculus,” Mathematical Gazette, vol. 56, no. 247, pp. 396-400, January, 1974. Article (CrossRef Link) 
[24] ER Love, "Fractional Derivatives of Imaginary Order," Journal of the London Mathematical Society, vol. s2-3, no. 2, pp. 241-259, February, 1971. Article (CrossRef Link)

[25] Wang Chengliang, Lan Libin, and Zhou Shangbo, "Adaptive fractional differential and its application to image texture enhancement,” Journal of Chongqing University, vol. 34, no. 2, pp. 32-37, February, 2011. Article (CrossRef Link)

[26] Huang Guo, Chen Qingli, and Xu Li, "Realization of adaptive image enhancement with variable fractional order differential," Journal of Shenyang University of Technology, vol. 34, no. 4, pp. 446-454, April, 2012. Article (CrossRef Link)

[27] $\mathrm{Pu}$ Yifei, Research on application of fractional calculus to latest signal analysis and processing, Sichuan University, 2006.

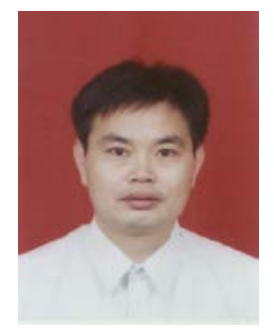

Jimin Yu received his Ph.D. in applied mathematics from Zhengzhou University in 2003. He is currently a professor with the key lab of industrial wireless networks and Networked Control of the Ministry of Education and the College of Automation, Chongqing University of Post and Telecommunications. His research interests include fractional order dynamic systems, numerical methods, and stability theory of functional equations.

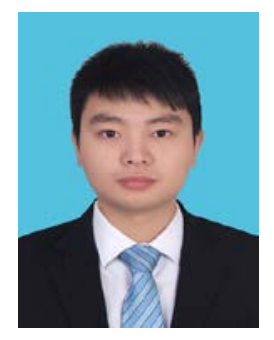

Lijian Tan received his B.S. degree in mechanical electrical engineering from Hubei University for Nationalities, China, in 2014. He is currently pursuing the M.S. degree in control engineering at the Chongqing University of Posts and Telecommunications. His research interests include image restoration and probe automatic positioning.

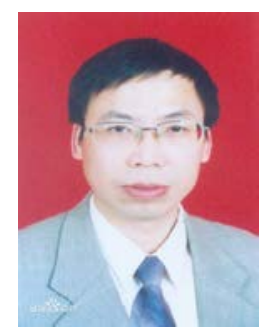

Shangbo Zhou received his BSc degree from Gangxi National College in 1985, MSc degree from Sichuan University in 1991, both in math, and PhD degree in circuit and system from Electronic Science and Technology University. From 1991 to 2000, he was with the Chongqing aerospace electronic and mechanical technology design research institute. Since 2003, he has been with the college of computer science and engineering of Chongqing University, where he is now a professor. His current research interests include artificial neural networks, physical engineering simulation, image processing and nonlinear dynamics. 


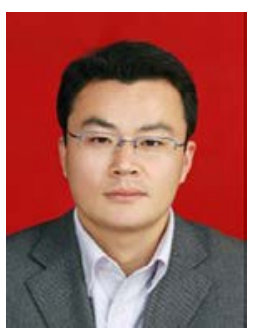

LipingWang received his BSc in Information Management and Information System from zhengzhou university, zhengzhou, China, in 2004. He worked as a teacher at college of Shengda economics trade and management of Zhengzhou university from 2004-2007. Received his master in technology of computer application from Chongqing university of posts and telecommunications, Chongqing, in 2010.he is currently working toward her $\mathrm{PhD}$ in college of computer science at Chongqing university. His research interests include image restoration, pattern recognition, and inverse problems.

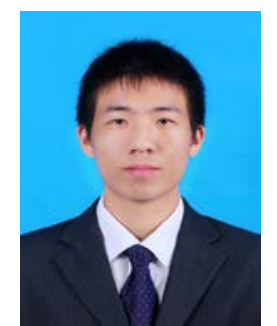

Chaomei Wang received his B.S. degree in automation from Hebei University of Engineering, China, in 2014. He is pursuing M.S. degree in control science and engineering at Chongqing University of Posts and Telecommunications. His interests focus on networked control theory. 